\title{
Isobrucein B
}

National Cancer Institute

\section{Source}

National Cancer Institute. Isobrucein B. NCI Thesaurus. Code C1774.

A quassinoid phytochemical isolated from the tropical plant Cedronia granatensis with potential antineoplastic and chemopreventive activities. (NCI04) 